\title{
The fractional Cubic Spline Interpolation without using the derivative values
}

\author{
Ahmad Reza Haghighi ${ }^{1}$ and Majid Roohi ${ }^{1}$ \\ ${ }^{1}$ Department of Mathematics, Urmia University of Technology, Urmia, Iran \\ ah.haghighi@gmail.com,majid.roohi67@gmail.com
}

\begin{abstract}
The paper introduces a function value based fraction of cubic spline interpolation, which is used for studying the curves and surfaces. The interpolation function has a simple and explicit mathematical representation, convenient both in practical application and in theoretical studies. It should be mentioned that the interpolating surfaces are $C^{1}$ in the interpolating region under the condition that the interpolation is only based on the function values. Moreover, properties and views are shown in matrix notation, and then the error is calculated.
\end{abstract}

2000 Mathematics Subject Classifications: 68U05, 65D05, 65D07, 65D18.

Keywords: Fractional spline; fractional interpolation: spline interpolation function, Peano Kernel theorem

\section{Introduction}

One of the most important cases in the field of computational mathematics that has always been taken into consideration and has a fundamental role in the numerical solution of mathematical problems is Interpolation issue. Among the current methods, Spline Interpolation is one of most important methods. Spline means a narrow and flexible strip (wood or metal) which is pliable. In the past, in order to design a ship or aircraft the curved strips was placed in a way that the desired crossing points must be flexible in use. For some physical reasons, if these curves are parameterized correctly, it will be in a multi-criteria and third degree also continuous second order derivative (Schumaker, 1981).

We know that for creating a normal spline polynomial function, we need first and second derivatives of function along with function values as interpolation data. They play an important role in the accuracy of the calculations. Besides, gaining the derivatives is not always possible. For example, in the case of describing the amount of rainfall in rainy areas or in some special geometrical cases (such as the design of industrial products etc.) gaining derived quantities is difficult and sometimes impossible. It means that, the major two problems in the splines is the general conditions and the dependency to the first and second derivative values (Duan et al., 1998; Duan et al., 2001; Duan et al., 2004).

In recent years the rational spline, especially the rational cubic spline with quadratic and cubic denominators, and its application to shape control, have received attention (Nielson, 1986; Sarfraz, 1992; Gregory et al., 1994; Sarfraz, 1994; Comninos, 2001; Sarfraz \& Hussain, 2006). Due to the stated issue, this paper introduces a fractional cubic spline interpolation function that is only based on function values, without the need for first and second derivatives. So, not only it has a simple mathematical display, but also it can be used for more accurate approximation to the desired data interpolation; with this property that the interpolation data do not change (Duan et al., 2000; Duan et al., 2006; Duan et al., 2007).

The uniqueness of interpolation for the required data, convert to uniqueness of the interpolation data and parameters. This fractional interpolation has the third degree numerator and a linear denominator (first degree).

In general, the relation that is presented here has continuous first order derivative. But the continuity of the second derivative depends on the parameters that make the Interpolation.

\section{The introduction of Interpolation}

\subsection{Interpolation}

Let $\left(t_{i}, f_{i}\right)$ be the given data $(i=1,2, \ldots, n, n+1)$, subject to $t_{i}$ is the value of function $f(t)$ in the $t_{i}$, which $f_{i} \in \mathbb{R}$ for $i=1,2, \ldots, n, n+1$. And, $=t_{0}<t_{1}<\cdots<t_{n}<t_{n+1}=b$, be the knot points of interval $[a, b]$. To expression of desired formula, First, we need to define some relations:

$$
\begin{aligned}
& p_{i}(t)=(1-\theta)^{3} \alpha_{i} f_{i}+\theta(1-\theta)^{2} v_{i}+\theta^{2}(1-\theta) w_{i}+\theta^{3} \beta_{i} f_{i+1}, \\
& q_{i}(t)=(1-\theta) \alpha_{i}+\theta \beta_{i},
\end{aligned}
$$


Where in the above equations $\theta=\frac{t-t_{i}}{h_{i}}$ and $h_{i}=t_{i+1}-t_{i}$. Also $v_{i}, w_{i}$ are offered, so that:

$$
\begin{aligned}
& v_{i}=\left(\alpha_{i}+\beta_{i}\right) f_{i}+\alpha_{i} f_{i+1}, \\
& w_{i}=\left(\alpha_{i}+2 \beta_{i}\right) f_{i+1}-h_{i} \beta_{i} \Delta_{i+1},
\end{aligned}
$$

Which $\Delta_{i}=\frac{I_{i+1}-f_{i}}{h_{i}}, \alpha_{i}>0$ and $\beta_{i}>0$.

Now by above relations, the desired Spline function is defined :

$$
\left.P(t)\right|_{\left[t_{i} t_{i+1}\right]}=\frac{p_{i}(t)}{q_{i}(t)}, i=1,2, \ldots, n-1,
$$

It is clear that $\mathrm{P}(\mathrm{t})$ is fractional interpolation with the cubic numerator and the linear denominator. Also this function in each sub-interval $\left[t_{i}, t_{i+1}\right]$ Have a continuous first order derivative. This is obtained definition of the functions $p_{i}(t)$ and $q_{i}(t)$. Therefore fractional interpolation function based on function values at $\left[t_{i}, t_{i+1}\right]$ and without having first and second derivatives, is made in (5). It can be easily shown that, the Interpolation function $P(t)$ in $(5)$, is unique and satisfy in : $P\left(t_{i}\right)=f_{i}, P\left(t_{i+1}\right)=f_{i+1}, P^{\prime}\left(t_{i+1}\right)=\Delta_{i+1}$ , (Duan et al., 1998).

Actually if suppose that

$$
P(t)=\frac{1}{(1-\theta) \alpha_{i}+\theta \beta_{i}}\left[a(1-\theta)^{3} \alpha_{i}+b \theta(1-\theta)^{2}+c t a^{2}(1-\theta)+\mathrm{d} \theta^{3} \beta_{i}\right]
$$

Then the relations $P\left(t_{i}\right)=f_{i}, P\left(t_{i+1}\right)=f_{i+1}, P^{\prime}\left(t_{i}\right)=\Delta_{i}$ and $P^{\prime}\left(t_{i+1}\right)=\Delta_{i+1}$ hold, if and only if $a=f_{i}, b=v_{i}, c=w_{i}$, $d=f_{i+1}$.

Obviously, the value of $P(t)$ depend on $\alpha_{i}$ and $\beta_{i}$. But if $\frac{\beta_{i}}{\alpha_{i}}$ is fixed, then $P(t)$ only depend on $\frac{\beta_{i}}{\alpha_{i}}$ (Duan et al., 1998; Duan et al., 2001; Duan et al., 2004). Now if knots have an equal distance, then (5) comes as:

$$
P(t)=\frac{1}{(1-\theta) \alpha_{i}+\theta \beta_{i}}\left[(1-\theta)^{2}\left(\alpha_{i}+\beta_{i} \theta\right) f_{i}+\theta\left(\alpha_{i}-\theta \alpha_{i}+3 \beta_{i} \theta-2 \beta_{i} \theta^{2}\right) f_{i+1}-\theta^{2}(1-\theta) \beta_{i} f_{i+2}\right]
$$

Moreover, if $\alpha_{i}=\beta_{i}$ then

$$
\mathrm{P}(\mathrm{t})=(1-\theta)^{2}(1+\theta) f_{i}+a\left(1-2 \theta-2 \theta^{2}\right) f_{i+1}-\theta^{2}(1-\theta) f_{i+2}, t \epsilon\left[t_{i}, t_{i+1}\right],-\theta^{2}(1-\theta) f_{i+2}, t \epsilon\left[t_{i}, t_{i+1}\right] \text {, }
$$

\subsection{The matrix mode of Interpolation}

Here we want to obtain the matrix of define Interpolation. Let knots have an equal distance, Means $h_{i}=h_{j}$ for each $i, j=1,2, \ldots, n$ , and consider $\alpha_{i}=\beta_{i}=$ constant. In this way $P(t)$ can be rewrite as the form of following matrix:

$$
\begin{aligned}
& P(t)=\omega_{0}\left(\theta, \alpha_{i}, \beta_{i}\right) f_{i}+\omega_{1}\left(\theta, \alpha_{i}, \beta_{i}\right) f_{i+1}+\omega_{2}\left(\theta, \alpha_{i}, \beta\right) f_{i+2} \\
& \omega_{0}\left(\theta, \alpha_{i}, \beta_{i}\right)=\frac{(1-\theta)^{2} \alpha_{i}+\theta(1-\theta)^{2} \beta_{i}}{(1-\theta) \alpha_{i}+\theta \beta_{i}}, \\
& \omega_{1}\left(\theta, \alpha_{i}, \beta_{i}\right)=\frac{\theta(1-\theta) \alpha_{i}+\theta^{2}(3-2 \theta) \beta}{(1-\theta) \alpha_{i}+\theta \beta_{i}}, \\
& \omega_{2}\left(\theta, \alpha_{i}, \beta_{i}\right)=\frac{-\theta^{2}(1-\theta)}{(1-\theta) \alpha_{i}+\theta \beta_{i}},
\end{aligned}
$$

Thus $\mathrm{P}(\mathrm{t})$ can be expressed as following equation:

$$
\begin{aligned}
& P(t)=A B, \\
& A=\left(\omega_{0}\left(\theta, \alpha_{i}, \beta_{i}\right), \omega_{1}\left(\theta, \alpha_{i}, \beta_{i}\right), \omega_{2}\left(\theta, \alpha_{i}, \beta_{i}\right)\right), \\
& B=\left(f_{i}, f_{i+1}, f_{i+2}\right)^{T},
\end{aligned}
$$

Now we want to study a particular case: 
Suppose that $\beta_{i}=1 . a_{r}$ are called as "weight coefficients integral" of the interpolation defined by(5), as follows:

$$
a_{r}=\int_{0}^{1} \omega_{r}\left(\theta, \alpha_{i}\right) d \theta, r=0,1,2
$$

The result is obtained immediately:

$$
\int_{x_{i}}^{x_{i+1}} P(t) d t=h_{i}\left(a_{0} f_{i}+a_{1} f_{i+1}+a_{2} f_{i+2}\right) \text {, }
$$

\subsection{Some of the features}

In this section We introduce some features of this Interpolation. Suppose that knots have an equal distance, and $\alpha_{i}, \beta_{i}$ have the constant values $\alpha$ and $\beta$ respectively. in the Interpolation relation that in (9) has been stated, $\omega_{\mathbf{r}}\left(\theta, \alpha_{i}, \beta_{i}\right)$ is considered as a basis for Interpolation. These basis features are:

\subsubsection{The first feature:}

In each sub-interval $\left[x_{i}, x_{i+1}\right]$ for every positive value of $\alpha$ and $\beta$, the total value of bases interpolation equals one, namely,

$$
\sum_{r=0}^{2} \omega_{r}\left(\theta, \alpha_{i}, \beta_{i}\right)=1 \text {, }
$$

\subsubsection{The second feature:}

In each sub-interval $\left[x_{i}, x_{i+1}\right]$ for every positive value of $\alpha$ and $\beta$, if in relation (5), $f_{i}=f_{i+1}=f_{i+2}$, then:

$$
\int_{x_{i}}^{x_{i+1}} P(t) d t=h_{i},
$$

So can write :

$$
a_{0}-a_{2}=\frac{1}{2}, a_{1}+2 a_{2}=\frac{1}{2}, \quad \sum_{r=0}^{2} a_{r}=1
$$

Since $a_{0}>0, a_{1}>0, a_{2}<0$ The following feature is available on integral weight coefficients:

\subsubsection{The third feature:}

In each sub-interval $\left[x_{i}, x_{i+1}\right]$, for every positive value of $\alpha$ and $\beta$, integral weight coefficients $a_{r}, r=0,1,2$, satisfy in:

$\frac{1}{4}<a_{0}<\frac{1}{2}, \frac{1}{2}<a_{1}<1,-\frac{1}{4}<a_{2}<0$,

In Table 1 the values of $a_{r}, r=0,1,2$, for $\alpha_{i}=\frac{1}{2}, 1,2$ is obtained.

Table 1. values of the integral weighted coefficients

\begin{tabular}{|c|c|c|c|}
\hline$\alpha_{i}$ & $\alpha_{0}$ & $\alpha_{1}$ & $\alpha_{2}$ \\
\hline$\frac{1}{2}$ & $\frac{19}{6}-4 \ln 2$ & $-\frac{29}{6}+8 \ln 2$ & $\frac{16}{6}-4 \ln 2$ \\
\hline 1 & $\frac{5}{12}$ & $\frac{2}{3}$ & $-\frac{1}{12}$ \\
\hline 2 & $-\frac{14}{6}+4 \ln 2$ & $\frac{37}{6}-8 \ln 2$ & $-\frac{17}{6}+4 \ln 2$ \\
\hline
\end{tabular}

\section{The error estimation}

To estimate error of fractional Cubic spline, we review the state that intervals are equal. Without loss of generality, we do it for the interval $\left[t_{i}, t_{i+1}\right]$.

Whenever $(t) e C^{2}[a, b]$, and $\mathrm{P}(\mathrm{t})$ be the fractional Interpolation function By using (Hussain et al., 2010) and the Peano - kernel Theorem:

$$
R(t)=f(t)-P(t)=\int_{t_{i}}^{t_{i+2}} f^{(2)}(\tau) R_{t}\left[(t-\tau)_{+}\right] d \tau,
$$


As:

$$
\begin{aligned}
& R_{t}\left[(t-\tau)_{+}\right]=\left\{\begin{array}{c}
(t-\tau)-\frac{1}{(1-\theta) \alpha_{i}+\theta \beta_{i}} \\
{\left[\theta\left(\alpha_{i}-\theta \alpha_{i}+3 \beta_{i} \theta-2 \beta_{i} \theta^{2}\right)\right.} \\
\left.\left(t_{i+1}-\tau\right)-\theta^{2}(1-\theta) \beta_{i}\left(t_{i+2}-\tau\right)\right], \\
t_{i}<\tau<t ; \\
-\frac{1}{(1-\theta) \alpha_{i}+\theta \beta_{i}}\left[\theta \left(\alpha_{i}-\theta \alpha_{i}\right.\right. \\
\left.+3 \beta_{i} \theta-2 \beta_{i} \theta^{2}\right)\left(t_{i+1}-\tau\right) \\
\left.-\theta^{2}(1-\theta) \beta_{i}\left(t_{i+2}-\tau\right)\right], \\
t<\tau<t_{i+1} ; \\
\frac{1}{(1-\theta) \alpha_{i}+\theta \beta_{i}} \theta^{2}(1-\theta) \beta_{i}\left(t_{i+2}-\tau\right), \\
t_{i+1}<\tau<t_{i+2} ;
\end{array}\right. \\
& =\left\{\begin{array}{l}
p(\tau), \quad t_{i}<\tau<t \\
q(\tau), \quad t<\tau<t_{i+1} \\
r(\tau) t_{i+1}<\tau<t_{i+2}
\end{array}\right.
\end{aligned}
$$

When $\mathrm{t} \epsilon\left[t_{i+1}, t_{i+2}\right]$ then $\mathrm{r}(\mathrm{t})>0$.

And the $q(t)$ can be written like this:

$$
q(\tau)=\frac{\left(\beta_{i} \theta^{3}+\left(\alpha_{i}-2 \beta_{i}\right) \theta^{2}-\theta \alpha_{i}\right)\left(t_{i+1}-\tau\right)}{+\theta^{2}(1-\theta) \beta_{i} h_{i}}
$$

Since

$$
q\left(t_{i}\right)=-h_{i} \theta \leq 0, q\left(t_{i+1}\right)=\frac{\theta^{2}(1-\theta) \beta_{i} h_{i}}{(1-\theta) \alpha_{i}+\theta \beta_{i}} \geq 0,
$$

Can be proved that the zero point $\tau^{*}$ in $q(t)$ satisfies:

$$
t_{i}<t<\tau^{*}=t_{i+1}+\frac{\theta^{2}(1-\theta) \beta_{i} h_{i}}{\beta_{i} \theta^{3}+\left(\alpha_{i}-2 \beta_{i}\right) \theta^{2}-\alpha_{i} \theta} t_{i+1},
$$

All of these show that:

$$
\begin{aligned}
& \text { If } \tau^{*}<\tau<t_{i+1} \text { then: } q(\tau) \geq 0 \\
& \text { If } t<\tau<\tau^{*} \text { then: } q(\tau) \leq 0
\end{aligned}
$$

Finally, for $p(\tau)$ since

$$
\begin{gathered}
p(\tau)=(t-\tau)+q(\tau), \\
p\left(t_{i}\right)=\left(t-t_{i}\right)+q\left(t_{i}\right)=0 \\
\text { and } \mathrm{p}(\mathrm{t})=\mathrm{q}(\mathrm{t})<0
\end{gathered}
$$

Thus for all $\tau \in\left[t_{i}, t\right], p(\tau) \leq 0$. 
So

$$
\begin{gathered}
|R[f]| \leq\left\|f^{(2)}\right\|\left[\int_{t_{i}}^{t}|p(\tau)| d \tau+\int_{t}^{\tau^{*}}|q(\tau)| d \tau \quad+\int_{\tau^{*}}^{t_{i+1}} q(\tau) d \tau+\int_{t_{i+1}}^{t_{i+2}} r(\tau) d \tau\right] \\
=\frac{h_{i}^{2}}{2}\left\|f^{(2)}\right\| \frac{w\left(\alpha_{i}, \beta_{i}, \theta\right)}{v\left(\alpha_{i}, \beta_{i}, \theta\right)},
\end{gathered}
$$

where

$$
\begin{aligned}
w\left(\alpha_{i}, \beta_{i}, \theta\right)= & \theta\left[\left(\alpha_{i} \beta_{i}-3 \beta_{i}^{2}\right) \theta^{4}+\left(7 \beta_{i}{ }^{2}-5 \alpha_{i} \beta_{i}+\alpha_{i}^{2}\right) \theta^{3}-\left(4 \beta_{i}^{2}-7 \alpha_{i} \beta_{i}+3 \alpha_{i}^{2}\right) \theta^{2}\right. \\
& \left.+3\left(\alpha_{i}{ }^{2}-\alpha_{i} \beta_{i}\right) \theta-\alpha_{i}^{2}\right] \\
v\left(\alpha_{i}, \beta_{i}, \theta\right)= & \left((1-\theta) \alpha_{i}+\beta_{i} \theta\right) \\
& \left(\beta_{i} \theta^{2}+\left(\alpha_{i}-2 \beta_{i}\right) \theta-\alpha_{i}\right)
\end{aligned}
$$

For given $\alpha_{i}$ and $\beta_{i}$ define:

$$
c_{i}=\max _{0<\theta<1} \frac{w\left(\alpha_{i} \beta_{i} \theta\right)}{v\left(\alpha_{i}, \beta_{i} \theta\right)},
$$

And call it the optimal error coefficient. Which immediately the following theorem is concluded:

\subsubsection{Theorem 1:}

suppose that $f(t) e C^{2}[a, b]$ and $\Delta=t_{0}<t_{1}<\cdots<t_{n}<t_{n+1}$ be nodes with equal distances. If $\alpha_{i}$ and be arbitrary and $P(t)$ be the desired interpolation function, then in $t \in\left[t_{i}, t_{i+1}\right]$ :

$$
|R[f]| \leq \frac{h_{i}{ }^{2}}{2}\left\|f^{(2)}(t)\right\|_{c_{i}}, \quad h_{i}=t_{i+1}-t_{i}
$$

The $c_{i}$ does not depend on to sub-interval $\left[t_{i}, t_{i+1}\right]$. But it just depend on to $\alpha_{i}$ And $\beta_{i}$ that shown in (7). Some values for the $c_{i}$ in fixed $\alpha_{i}$ and Some values for the $c_{i}$ in fixed $\beta_{i}$ shown in Table 2 and Table 3 respectively. from these values can be realized that the $c_{i}$ are bounded for each value of $\alpha_{i}$ and $\beta_{i}$. In fact the following theorem can be proved:

\subsubsection{Theorem 2:}

for each value of $\alpha_{i}$ and $\beta_{i}, c_{i}$ is bounded and:

$$
\frac{1}{4} \leq c_{i}<\max _{0 \leq \theta \leq 1} \frac{3 \theta^{3}-7 \theta^{2}+4 \theta}{2-\theta}=\quad 0.42330428
$$

Table 2. Some values for the c_i in fixed a_(i)

\begin{tabular}{|l|l|l|l||}
\hline $\mathrm{i}$ & $\alpha_{i}$ & $\beta_{i}$ & $\mathrm{c}_{i}$ \\
\hline \hline 1 & 1 & 1 & 0.301805 \\
\hline 2 & 1 & 1.1 & 0.306085 \\
\hline 3 & 1 & 1.2 & 0.310069 \\
\hline 4 & 1 & 1.5 & 0.320499 \\
\hline 5 & 1 & 2 & 0.334042 \\
\hline 6 & 1 & 3 & 0.352379 \\
\hline 7 & 1 & 5 & 0.372697 \\
\hline 8 & 1 & 10 & 0.393554 \\
\hline 9 & 1 & 20 & 0.406906 \\
\hline 10 & 1 & 50 & 0.416295 \\
\hline 11 & 1 & 100 & 0.419715 \\
\hline 12 & 1 & 1000 & 0.422937 \\
\hline
\end{tabular}

Table 3. Some values for the $c_{-} i$ in fixed $\beta \_(i)$

\begin{tabular}{|l|l|l|l|}
\hline \multicolumn{1}{|c|}{$\mathrm{i}$} & $\alpha_{i}$ & $\beta_{i}$ & $\mathrm{c}_{i}$ \\
\hline \hline 1 & 1 & 1 & 0.301805 \\
\hline 2 & 1.1 & 1 & 0.297633 \\
\hline 3 & 1.2 & 1 & 0.293937 \\
\hline 4 & 1.5 & 1 & 0.285055 \\
\hline 5 & 2 & 1 & 0.275151 \\
\hline 6 & 3 & 1 & 0.264602 \\
\hline 7 & 5 & 1 & 0.256627 \\
\hline 8 & 10 & 1 & 0.252003 \\
\hline 9 & 20 & 1 & 0.250556 \\
\hline 10 & 50 & 1 & 0.250095 \\
\hline 11 & 100 & 1 & 0.250024 \\
\hline 12 & 1000 & 1 & 0.250000 \\
\hline
\end{tabular}




\section{Numerical example}

Suppose that $f(x)=\sin ^{3}(x)+\cos ^{4}(x)$ and let $h_{i}=0.2, x_{i}=\frac{i}{5}-3.5$ for $i=1,2, \ldots, 20$. If $P(x)$ be the interpolation function of $f(x)$ which introduce in (5); Figs.1-4 show the approximation of $f(x)$ by the function $P(x)$.

Fig.1. Interpolation of

$f(x)=\sin ^{3}(x)+\cos ^{4}(x)$ for $a_{i}=10$ and $\beta_{i}=1$

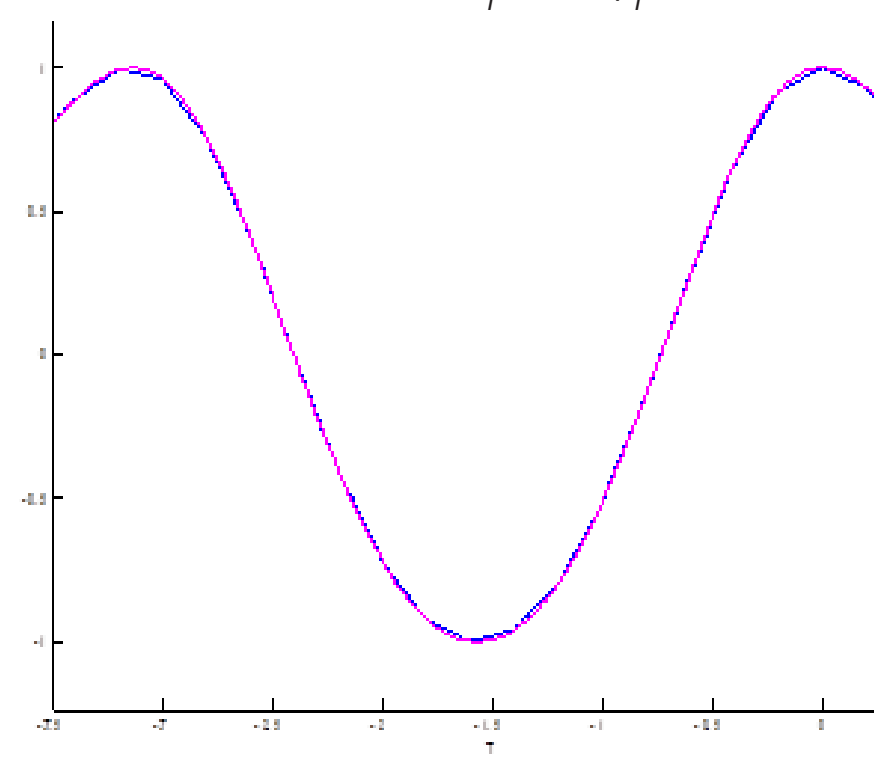

Fig.3. Interpolation of

$f(x)=\sin ^{3}(x)+\cos ^{4}(x)$ for $a_{i}=1$ and $\beta_{i}=10$

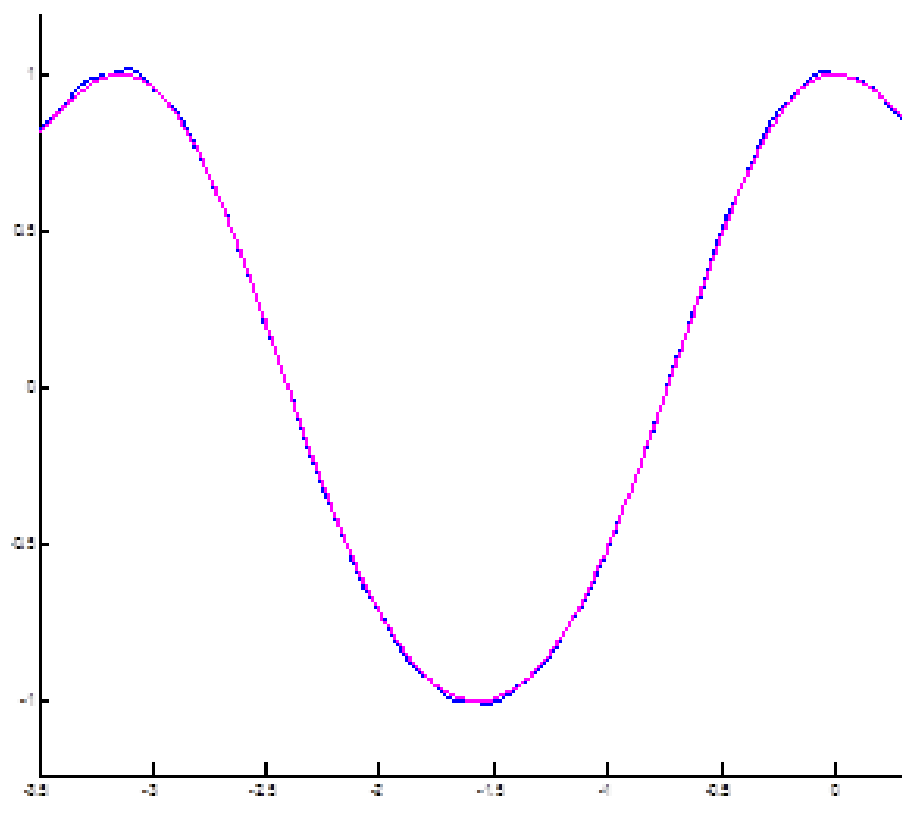

Fig.2. Interpolation of

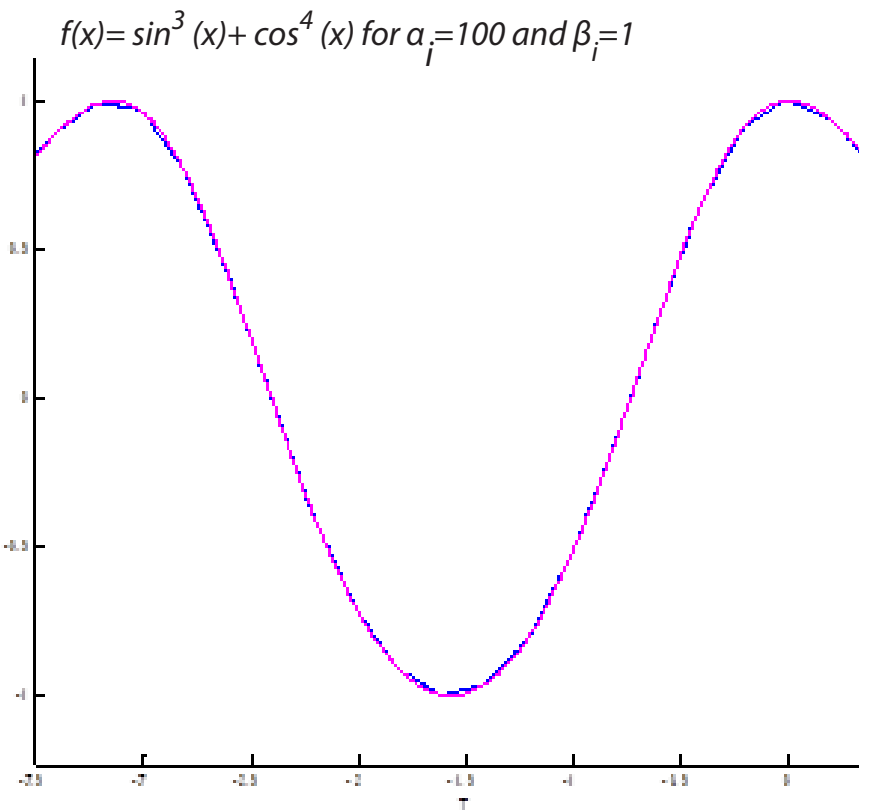

Fig.4. Interpolation of the function

$f(x)=\sin ^{3}(x)+\cos ^{4}(x)$ for $a_{i}=1$ and $\beta_{i}=1$

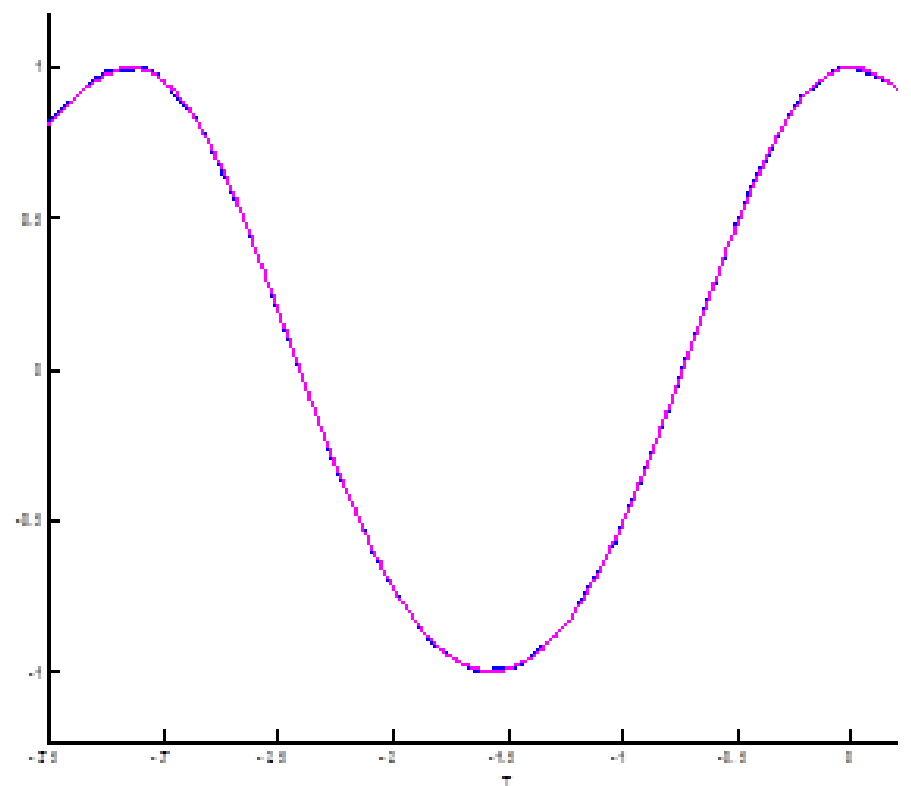

The figures reveal that the desired approximation for the $\alpha_{i}=1$ and $\beta_{i}=1$. Fig.4 exhibits more accurate approximations than the Figs. $1 \& 2$ and Fig. 3 for the $\alpha_{i}=10, \beta_{i}=1$ and $\alpha_{i}=10, \beta_{i}=1$ and $\alpha_{i}=1, \beta_{i}=10$ respectively. 


\section{Conclusions}

The fractional Interpolation presented in this article is only based on function values and parameters $\alpha_{i}$ and $\beta_{i}$, without using function derive value. This kind of interpolation not only has a simple and explicit formulation, but also used in theoretical studies and software methods. In addition, the presented relation can be displayed as a matrix relation. The $\alpha_{i}$ and $\beta_{i}$ are arbitrary in this regard, and must be equal or greater than zero, but both should not be zero at the same time; besides, by assigning different values to them, interpolation approximations can be improved. The relation presented in this paper has a tiny and bounded error coefficient that shows approximation of interpolation is accurate.

\section{References}

1 Comninos (2001) "An interpolating piecewise bicubic surface with shape parameters.” Comput. Graph. 25 , 463-481.

2 Duan Q, Djidjeli K, Price WG and Twizell EH (2000) "Weighted rational cubic spline interpolation and its approximation.” J. Comput. Appl. Math. 117.121-135.

3• Duan Q, Djidjeli K, Price WG and Twizell EH (1998) "Rational cubic spline based on function values.” Comput. Graph. 22, 479-486.

4- Duan Q, Liu A and Cheng F (2001) “Constrained rational cubic spline and its application.” J. Comput. Math. 19 143-150.

5• Duan Q, Wang L and Twizell EH (2004) “A new bivariate rational interpolation based on function values." Inform. Sci. 166, 181-191.

6• Duan Q, Zhang H, Zhang Y and Twizell EH (2007) “Error estimation of a kind of rational spline.” J. Comput. Appl. Math. 200(1), $1-11$

7• Duan Q, Zhang Y and Twizell EH (2006) “A bivariate rational interpolation and the properties.” Appl. Math. Comput. 179,190199.

8• Gregory, JA, Sarfraz M and Yuen PK (1994) "Interactive curve design using C 2 rational spline.” Comput. Graph.18, 153-159.

9• Hussain MZ, Sarfraz M and Hussain M (2010) "Scientific data visualization with shape preserving $C^{l}$ rational cubic interpolation." Eur.J.Pure \& Appl.Math. 3 (2), 194-212.

10• Nielson GM (1986 ) "Rectangular v-splines.” IEEE comp. Graph. Appl. 6, 35- 40.

11 Sarfraz M (1992) 'Convexity preserving piecewise rational interpolation for planar curves.” Bull. Kor. Math. Soc. 29, 193-200.

12. Sarfraz M and Hussain M Z, (2006) “Data visualization using spline interpolation.' J. Comput. Appl. Math. 189, 513-525.

13• Sarfraz M (1994) 'Cubic spline curves with shape control.” Comp \& Graph. 18(5), 707-713.

14• Schumaker LL (1981) "Spline functions: Basic theory." Cambridge Mathematical Library, Wiley, New York. 